\title{
Invasive pulmonary aspergillosis complicating severe influenza:
}

\section{epidemiology, diagnosis and treatment}

Lore Vanderbeke $^{1,2}$, Isabel Spriet ${ }^{3,4}$, Christine Breynaert $^{5}$, Bart J.A. Rijnders ${ }^{8}$, Paul E. Verweij ${ }^{9,10}$, Joost Wauters $^{2,11}$

${ }^{1} \mathrm{KU}$ Leuven Department of Microbiology and Immunology, Laboratory of Clinical Bacteriology and Mycology, Leuven, Belgium

${ }^{2}$ University Hospitals Leuven, Department of General Internal Medicine, Medical Intensive Care Unit, Leuven, Belgium

${ }^{3}$ KU Leuven Department of Pharmaceutical and Pharmacological Sciences, Laboratory of Clinical Pharmacology and Pharmacotherapy, Leuven, Belgium

${ }^{4}$ University Hospitals Leuven, Department of Pharmacy, Leuven, Belgium

${ }^{5} \mathrm{KU}$ Leuven Department of Microbiology and Immunology, Laboratory of Clinical Immunology, Leuven, Belgium ${ }^{6}$ University Hospitals Leuven, Department of Hematology, Leuven, Belgium

${ }^{7}$ University Hospitals Leuven, Department of Laboratory Medicine and National Reference Center for Mycosis, Leuven, Belgium.

${ }^{8}$ Department of Internal Medicine, section of Infectious Diseases, Erasmus University Medical Center, Rotterdam, The Netherlands.

${ }^{9}$ Department of Medical Microbiology, Radboud University Medical Centre, Nijmegen, The Netherlands ${ }^{10}$ Centre of Expertise in Mycology, Radboudumc/CWZ, Nijmegen, The Netherlands

${ }^{11} \mathrm{KU}$ Leuven Department of Microbiology and Immunology, Laboratory for Clinical Infectious and Inflammatory Disorders, Leuven, Belgium

\section{Correspondence to}

Lore Vanderbeke, MD, Department of General Internal Medicine, University Hospitals Leuven, Herestraat 49, Leuven, Belgium. Tel: +3216 345 145; e-mail: lore.vanderbeke@uzleuven.be 


\section{Purpose of review}

Bacterial superinfection of critically ill influenza patients is well-known, but in recent years, more and more reports describe invasive aspergillosis as a frequent complication as well. This review summarizes the available literature on the association of invasive pulmonary aspergillosis (IPA) with severe influenza (influenza-associated aspergillosis, IAA), including epidemiology, diagnostic approaches and treatment options.

\section{Recent findings}

Though IPA typically develops in immunodeficient patients, non-classically immunocompromised patients such as critically ill influenza patients are at high-risk for IPA as well. The morbidity and mortality of IPA in these patients is high and in the majority of them, the onset occurs early after intensive care unit admission. Currently, standard of care (SOC) consists of close follow-up of these critically ill influenza patients with high diagnostic awareness for IPA. As soon as there is clinical, mycological or radiological suspicion for IAA, antifungal azole-based therapy (e.g. voriconazole) is initiated, in combination with therapeutic drug monitoring (TDM). Antifungal treatment regimens should reflect local epidemiology of azole-resistant Aspergillus species and should be adjusted to clinical evolution. TDM is necessary as azoles like voriconazole are characterized by nonlinear pharmacokinetics, especially in critically ill patients.

\section{Summary}

In light of the frequency, morbidity and mortality associated with influenza-associated aspergillosis in the intensive care unit (ICU), a high awareness of the diagnosis and prompt initiation of antifungal therapy is required. Further studies are needed to evaluate the incidence of IAA in a prospective multi-centric manner, to elucidate contributing host-derived factors to the pathogenesis of this superinfection, to further delineate the population at risk and to identify the preferred diagnostic and management strategy as well as the role of prophylaxis.

\section{Key words}

influenza, ICU, aspergillosis, antifungals, Aspergillus fumigatus 


\section{Introduction}

Worldwide, 3-5 million people develop severe influenza infection every year, leading to 50,000100,000 deaths annually in the European Union and the USA. Of the hospitalised patients $5-10 \%$ need ICU admission. These patients initially present with typical influenza symptoms, but develop rapidly evolving respiratory deterioration potentially leading to acute respiratory distress syndrome (ARDS) associated with high mortality [1,2]. Bacterial superinfection, mainly with Streptococcus pneumoniae and Staphylococcus aureus, is a well-known complication of severe influenza and its pathophysiology has been widely studied [3]. In recent years however an increasing number of publications on influenza-associated aspergillosis is reported and the largest retrospective case study on the subject was recently published [ $\mathbf{4}^{\mathbf{W}}$ ]. This review will focus on epidemiological data from published cases of IAA up to June 2018 as well as on new insights related to the diagnosis and treatment of this infection in the ICU-admitted patient. A brief note on the pathogenesis and suggested topics for further research are presented as well. References were identified using PubMed and Embase searches (January 1950 - June 2018) of both English and foreign language literature using the search terms "influenza" and "aspergillus", "aspergillosis" or "invasive aspergillosis". References of identified case reports and case series were reviewed to identify additional articles for inclusion.

\section{Epidemiology}

There is a remarkable increase in published cases over time: the first report of probable IAA dates back to 1952 , with up to 6 case reports per decade thereafter. A sharp increase in reported cases occurred after the 2009 H1N1 influenza pandemic (Figure 1). Whether this is associated with an increasing trend of Aspergillus superinfection or with improved diagnostics remains unclear though the latter is much more likely. Up to June 2018128 IAA cases have been published, which are summarized in table 1 [5$\left.34,3^{\mathbf{}}, 36-50,51^{\bullet}, 52^{-}, 53-54\right]$. Most cases had at least one underlying medical condition, yet $28 \%$ were reported as previously healthy. The most frequently observed underlying conditions were the use of one or more immunosuppressive drugs for a variety of underlying diseases $(n=32,25 \%)$, 
haematological malignancy $(n=19,15 \%)$ and diabetes $(n=19,15 \%)$. Only $9 \%(n=11)$ of patients were reported to take corticosteroid (CS) therapy before influenza diagnosis, while $48 \%$ ( $n=61$ ) were treated with CS during hospitalisation. Antiviral and antifungal therapy was administered in the majority of cases (69\% and $89 \%$ respectively). Most IAA cases were found in patients with influenza A infection ( $n=111,87 \%)$, predominantly associated with H1N1 virus. IAA diagnosis was frequently made early after ICU admission (median of 5 days after admission; IQR: 2 - 11.5). Diagnosis of IAA was largely based on culture ( $n=89,70 \%)$. Galactomannan (GM) antigen was reported positive in serum in half of the patients ( $n=60,47 \%)$ and in bronchoalveolar lavage (BAL) fluid in one third of the patients ( $n=39$, 31\%). Bronchoscopy revealed fungal tracheobronchitis in 19 patients (15\%). These results need to be interpreted with caution, because not all diagnostic techniques were available in every medical centre and case definitions differed. Therefore, these numbers in no way reflect the sensitivity or specificity of these tests. IAA was proven through pathological examination in 40 patients (31\%), of which 19 (48\%) were proven only at autopsy. Most patients required mechanical ventilation $(n=100,78 \%)$ and overall mortality was $57 \%(n=73)$. Though there may be regional differences in prevalence, reports have occurred in all world continents, except South-America and Antarctica (Table 2).

Additionally, four recent large retrospective studies in ICU-patients need to be mentioned. In the first of these four reports, Martin-Loeches et al studied the occurrence of superinfection in 2901 critically ill influenza patients from a large number of ICUs in Spain between 2009 and 2015. In this dataset, $16.6 \%(n=482)$ had a superinfection and of those Aspergillus was found in $7.2 \%(n=35)$ in the two days after ICU admission [55]. The second report is a retrospective cohort of 134 patients on extracorporeal membrane oxygenation (ECMO) in a tertiary care centre in the UK during 2012-2016. Ten of them had evidence of an invasive Aspergillus infection and influenza A was an independent risk factor for IPA (HR 11.4, $95 \% \mathrm{Cl} 1.97-65.86$ ) [56]. In the third report, Cavayas et al. studied the risk factors for fungal infection in an international database of 2,129 ECMO patients. Here again, influenza was an independent risk factor for IAA in ECMO patients (OR 2.48)[57"-]. Finally, in a paper on 432 patients with influenza admitted to seven ICUs in the Netherlands and Belgium over a period of 7 years, $19 \%$ 
$(n=83)$ were diagnosed with invasive aspergillosis. Of the 315 influenza patients without a typical host factor that would put them at risk for invasive aspergillosis, the incidence remained high at $14 \%$. A comparison of influenza patients with severe non-influenza pneumonia patients allowed for the identification of influenza as an independent risk factor for IPA (aOR 5.2, 95\% Cl 2.6-10.3). Moreover, IAA was diagnosed very early after ICU admission (median of 3 days after admission), which highlights the need for a structured and prompt diagnostic approach [4"].

\section{Diagnosis}

Microbiological detection of influenza can be performed on nasopharyngeal or lower respiratory tract samples. Nucleic acid testing is both rapid and highly sensitive, making it the preferential detection method in most hospitals. Viral culture has similar sensitivity, yet is time- and labour intensive. Direct antibody testing, though clearly less sensitive, is rapid and does not require specialised skills [58]. Sampling of the lower respiratory tract (LRT) has a higher diagnostic yield and positivity in LRT is associated with worse outcome in critically ill influenza patients [59].

Diagnosing IPA is less straightforward. Histological identification of acute-angle branching septated hyphae invading lung tissue is needed to prove a diagnosis of IPA. Lung biopsy is however rarely performed in critically ill patients because of the risk of respiratory complications and bleeding.

A recent national survey in the Netherlands showed a hospital mortality of $61 \%$ in 23 ICU patients with IAA. Patients that survived had received antifungal therapy at a median of two days after influenza diagnosis, compared with nine days in patients that had died, indicating that early diagnosis and treatment are critical [51"]. Awareness of invasive aspergillosis as a potential cause of secondary infection is important as IAA occurs in non-typical host groups and characteristic CT images such as 'halo-sign' might be absent. Therefore any respiratory specimen harbouring Aspergillus species in culture or a positive GM should not be disregarded in ICU-patients with severe influenza. A bronchoscopy with BAL appears the preferred diagnostic approach as the performance of GM detection and culture showed good sensitivity in IAA, i.e. $94 \%$ and $78 \%$ respectively [51"]. As up to $15 \%$ 
of patients may develop Aspergillus tracheobronchitis, the presence of plaques in the trachea or bronchi should be noted during bronchoscopy, which is recommended by the IDSA [60]. As radiological manifestations of tracheobronchitis are subtle or may be absent, visualization of plaques is regarded the best way to diagnose this condition [61].

In addition to GM detection in BAL, serum GM was also positive in 64 to $71 \%$ of IAA-patients, which is a high sensitivity considering that most patients were non-neutropenic $\left[4^{\mathbf{M}}, 51^{\mathbf{M}}\right]$. A positive serum GM increases the probability of IAA and helps to distinguish invasive infection from respiratory colonization. Any positive serum GM in a patient with severe influenza therefore should prompt immediate antifungal treatment, even if pre-existing risk factors are absent. The logistics of GM detection however might cause diagnostic delay as in many centres GM-testing is performed only once or twice a week. Recently lateral flow device (LFD) tests became available for detection of Aspergillus antigens in serum and BAL. The main advantage of the LFD assay is that results are available within 30 minutes, although the concentration of the antigen is not quantified [62].

In geographical regions in which azole resistance has been described, the detection of resistance should be pursued in clinically relevant isolates [63]. As mixed infections (e.g. azole-susceptible and azole-resistant co-infection) have been reported in patients with IAA, multiple colonies should be investigated in culture-positive patients. Screening through agar-based systems such as the VIPcheck ${ }^{\mathrm{TM}}$ test enables detection of resistance within 24 to $48 \mathrm{hrs}$. If the screening test indicates resistance, MICtesting can be performed [64]. Culture and phenotypic resistance screening and MIC-testing can take up to a week or longer and therefore may be too late to guide antifungal therapy. Indeed a recent study in culture-positive patients with invasive aspergillosis indicated that initial voriconazole therapy corresponded with a $27 \%$ higher mortality in patients with voriconazole-resistant A. fumigatus compared with patients with voriconazole-susceptible infection. Despite resistance screening and MICtesting the median time to change to appropriate antifungal therapy was 10 days, indicating that resistance information should be available earlier to prevent mortality [65]. In culture negative patients resistance-PCR can be performed directly on clinical specimens. Two commercial PCR-tests 
are available (MycoGenie and AsperGenius), which detect one or two common resistance markers. Generally, the assays show acceptable sensitivity when done on BAL fluid and results are available within a working day $[66,67]$. In culture positive patients, these PCR-tests can also be performed directly on a suspension of multiple colonies with excellent sensitivity and with additional advantage that, compared with phenotypic resistance testings, the PCR result will be available the same day. The limitation is that resistance mechanisms other than the mutations included in the PCR will go unnoticed.

Given the rapid disease progression in IAA a diagnostic pathway could include early BAL, followed by LFD and, if positive, Aspergillus and resistance PCR. Although such a strategy still needs to be validated, IAA diagnosis could be made within 48 hours including information regarding the presence of resistance markers.

\section{Treatment}

In this section, an overview of current knowledge on the pharmacological treatment, comprising antivirals, antifungals and corticosteroid therapy is given. Respiratory organ support techniques, such as prone positioning and ECMO contribute to improved survival, but fall outside the scope of this review [68].

\section{Antiviral therapy}

Neuraminidase inhibitors (NAls) (oseltamivir, zanamivir and very recently peramivir) form the only drug class that are licensed and recommended for the treatment of influenza infection in Europe $[69,70]$. Current evidence supports the use of oseltamivir in severe influenza, if administered early. Timely administration has indeed shown reduced ICU length of stay, reduced mechanical ventilation days and improved survival in several cohorts of ICU patients [71-73]. During the pandemic 2009 H1N1 infection, the World Health Organization listed oseltamivir as a 'core drug' and advised to treat with oseltamivir at a higher dose (150 mg twice daily instead of $75 \mathrm{mg}$ twice daily) and for longer duration (standard treatment duration being 5 days) in severely ill patients (1). These recommendations are 
based on animal models, with only limited evidence for benefit in human cohorts [74]. No prospective studies on optimal duration of treatment in critically ill influenza patients exist, yet extended treatment duration with oseltamivir was associated with a trend towards improved mortality in a retrospective analysis of 19,892 adult ICU patients [75]. Regarding the optimal dosing of oseltamivir in severe influenza patients, the evidence is inconclusive as well. The only three prospective studies comparing standard and high-dose oseltamivir in hospitalised patients show conflicting evidence on improved virological clearance without obvious clinical benefit of high-dose therapy [76-78]. Additional retrospective studies corroborate the absence of effect on clinical outcome such as ICU length-of-stay or mortality $\left[7^{\boldsymbol{M}}, 80\right]$. In light of this conflicting data, the World Health Organisation downgraded oseltamivir on its drug list [81]. Furthermore, pharmacokinetic analysis in critically ill pandemic H1N1 influenza virus patients showed that enteric absorption of oseltamivir at standard dose was adequate, resulting in trough levels and area under the curve of the active carboxylate metabolite well above the $50 \%$ maximal inhibitory concentration of the influenza virus [82].

Dosing of oseltamivir in critically ill patients should be adapted to the provided organ-support. Despite the fact that ECMO can alter pharmacokinetics, by increasing the volume of distribution and sequestration of drugs in the circuit among others, no dose adjustment for oseltamivir seems warranted based on current reports [83-85]. Dose reduction to $75 \mathrm{mg}$ once daily is however recommended in patients on renal replacement therapy (RRT), depending on type of RRT and patient characteristics $[83,84,86-88]$. Recommendations are based on limited available evidence in small patient populations, thus better evidence is clearly required. Current research is focusing on new NAls (such as peramivir), new antiviral drug classes (e.g. endonuclease inhibitors) and the role of combination therapy $[89,90]$.

\section{Antifungal therapy}

Voriconazole, the current gold standard therapy for IPA according to ECIL/ESCMID-ECMM-ERS/IDSA guidelines, led to a higher overall survival compared to conventional amphotericin $B[60,63,91]$. The intravenous formulation is used in ICU setting, to avoid erratic absorption and to warrant therapeutic 
plasma levels. Although voriconazole has earned its merit, its use is hindered by non-linear pharmacokinetics, risk for neuro- and hepatotoxicity and frequent involvement in drug-drug interactions. These factors underscore the need for therapeutic drug monitoring (TDM), aiming at trough levels of 2-5.5 mg/L $[60,63]$. Especially during ECMO, TDM is warranted since voriconazole sequestration and subsequent saturation of binding sites of the ECMO circuit can significantly alter drug levels [92,93]. Moreover, multiple drugs are known to interact with voriconazole based at the level of cytochrome P450 metabolizing enzymes [94,95]. Next to these classic CYP450 mediated interactions, also other interactions have been described in literature. A new and clinically relevant interaction was described between flucloxacillin and voriconazole showing subtherapeutic levels of voriconazole in 11 of 20 patients treated simultaneously with both drugs [96"].

Isavuconazole is a new extended-spectrum triazole that can be administered as a prodrug via oral or intravenous formulation. Its longer half-life compared to voriconazole allows for once daily dosing after an initial two-day period of three times daily loading. Compared to voriconazole, isavuconazole has a more favourable profile, regarding safety and spectrum (including some of the Mucorales) while being non-inferior for primary treatment of suspected invasive mould disease [97-99]. This is reflected in current treatment guidelines; isavuconazole has the same level of evidence as voriconazole $[60,63]$. More clinical data are needed to provide insight in the efficacy and safety of isavuconazole at the ICU, the need for therapeutic drug monitoring and optimal dosing in patients on ECMO $[100,101]$. For many years, the polyene conventional amphotericin B was the mainstay therapy for invasive mould infection and its liposomal formulations have been associated with a better safety profile. However, its use is currently limited to patients in which voriconazole is contra-indicated, e.g. in case of hepatic insufficiency or in cases of suspected or proven azole-resistance. Importantly, with the widespread use of azoles in the environment, resistance has been observed worldwide, with resistance rates of up to $26 \%$ in certain Dutch ICUs $\left[102^{\prime}, 103\right]$. Recent guidelines recommend to start voriconazoleechinocandin combination or liposomal amphotericin B as initial therapy in regions with environmental resistance rates of $>10 \%$ [63]. If susceptibility testing demonstrates azole-susceptible Aspergillus 
(voriconazole $\mathrm{MIC} \leq 1 \mathrm{mg} / \mathrm{L}$ ) voriconazole monotherapy is recommended, while in azole-resistant Aspergillus (voriconazole MIC $>2 \mathrm{mg} / \mathrm{L}$ ) ongoing therapy should consist of an antifungal drug to which the recovered species is susceptible $[104,105]$. Furthermore, combination therapy is recommended in IDSA guidelines for salvage therapy [60]. Regardless of the initial treatment regimen, timely administration of appropriate antifungal therapy is the single most crucial intervention to improve outcome, therefore initiation of treatment at suspicion of aspergillosis is advocated $[60,106]$. Primary prophylaxis with posaconazole is currently only recommended in high-risk patient groups (i.e. acute myelogenous leukaemia or myelodysplastic syndrome receiving induction chemotherapy) [63]. The effect of prophylactic antifungal administration in critically ill influenza patients requires further investigation.

\section{Corticosteroids}

CS treatment is frequently used in the ICU, both as adjunctive treatment in septic shock and in the late phase of ARDS, although its value in septic shock remains a matter of debate [107-109]. Moreover, guidelines on treatment of severe pneumonia are being reviewed, considering CS treatment early in the disease course to improve morbidity and mortality $\left[110^{*}, 111\right]$. Influenza pneumonia is however deemed an exception, based on low quality evidence showing an association between CS and prolonged viral shedding with increased risk of mortality [1,112-114]. The available evidence on the value of CS in patients with influenza argues against its use as long as data from a prospective randomized clinical trial are lacking. Additionally, CS were an independent risk factor for the development of IPA in ICU patients in general but very recently also in patients admitted with influenza

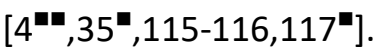

\section{Pathogenesis}

The underlying mechanisms that render influenza-patients prone to develop IAA remain to be elucidated and is mainly derived from studies regarding influenza-bacterial superinfection. Influenza induces damage to the respiratory tract epithelial lining, interferes with normal mucociliary clearing and in this way can provide a gateway for Aspergillus infection. Moreover, immunological host 
responses are altered in the setting of severe influenza, with dysregulation of Th cell differentiation and impaired cell-mediated immunity $[3,118]$. Additionally, treatment strategies in the ICU may enhance susceptibility to secondary aspergillosis. CS are well-known to downregulate innate and more importantly adaptive immunity, contributing to IPA development. In vitro, neuraminidase inhibitors are able to diminish viral-bacterial synergism that may contribute to severe influenza lethality [119]. The opposite might be true for the influenza-aspergillosis association as initial in vivo experiments in immunodeficient mice show more severe IPA development in cortisone-treated animals exposed to oseltamivir compared to controls, a difference that was not observed in neutropenic mice [120"']. Further research is needed to clarify the multiple contributing factors in the pathophysiology of this disease.

\section{Conclusion and perspectives}

IAA is a frequent and severe complication of influenza in ICU, occurring early after admission. High diagnostic awareness, incorporating a multiple biomarker-diagnostic strategy, and prompt initiation of antifungal treatment are crucial to improve outcome. We recommend considering Aspergillus infection in all ICU-patients with severe influenza, irrespective of previous medical history. Although the optimal diagnostic work-up remains to be validated, early bronchoscopy and BAL with the use of biomarkers appears to be important to diagnose IAA as early as possible. If initial assessments are negative for aspergillosis, this strategy should be repeated in case of respiratory deterioration. Further studies are needed to provide better epidemiological insights, currently a multi-centre observation trial is ongoing (NCT03391492), to investigate the potential of antifungal prophylaxis (NCT03378479) and to elucidate the pathophysiology of this superinfection. 


\section{Bullet points}

- Influenza associated aspergillosis is a frequent and severe superinfection in the intensive care unit.

- IAA may occur in any patient and shows a rapid disease progression with a high mortality.

- A broader awareness of this complication and prompt antifungal treatment initiation are crucial.

- Detailed knowledge about the underlying pathophysiological mechanisms and better risk assessment scores are urgently needed.

\section{Acknowledgements}

Lore Vanderbeke received internal funding from KU Leuven while working on the present review article, and at the time of publication is a PhD fellow of the Research Foundation - Flanders (FWO). Isabel Spriet and Christine Breynaert are supported by the Clinical Research Fund, University Hospitals Leuven.

Joost Wauters is a senior clinical investigator, Research Foundation - Flanders (FWO). 
Figure 1: Overview of IAA cases and publications in literature over time (1952-2017 period) [534,35",36-50,51",52",53-54].

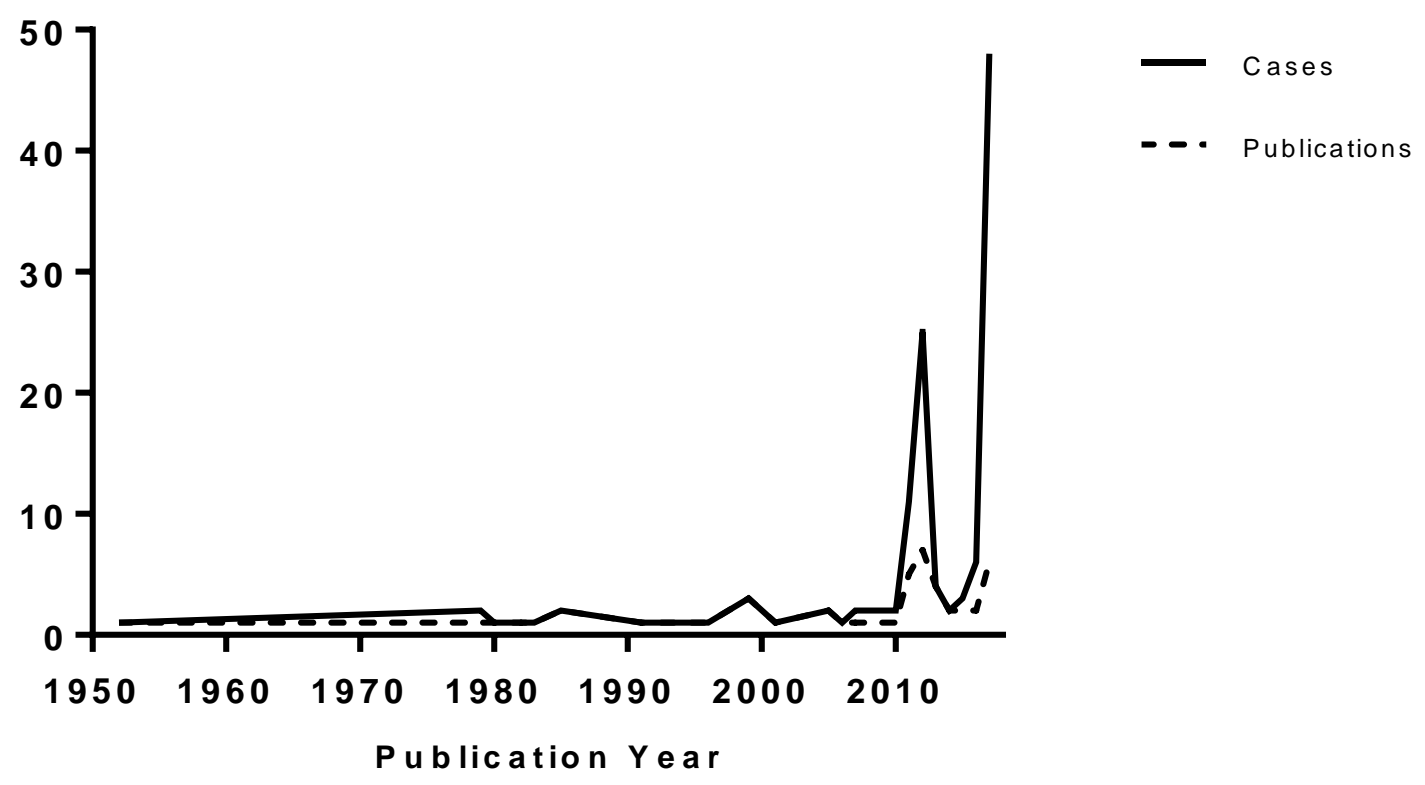


Table 1: Summary of 128 cases of influenza-associated aspergillosis: literature review of cases and

case series [5-34,35",36-50,51",52",53-54].

BAL: bronchoalveolar lavage; COPD: chronic obstructive pulmonary disease; CT: computed tomography; ECMO: extracorporeal membrane oxygenation; EORTC: European organisation for research and treatment of cancer; IQR: interquartile range; PCR: polymerase chain reaction.

* Since multiple diagnostic techniques were employed in a majority of reported cases, the total percentage in the diagnosis category adds up to more than a $100 \%$. All subcategories are listed relative to the total number of 128 cases, even though not every technique was employed in every case.

a Percentages relative to total number of influenza A cases reported.

${ }^{b}$ Percentages relative to total number of CT scans reported.

'Time from influenza diagnosis to aspergillosis diagnosis only reported in 70 reports.

dPercentages relative to total number of antiviral use.

eTime from admission to death only reported in 44 reports.

\begin{tabular}{|c|c|}
\hline CHARACTERISTIC & $\begin{array}{l}\text { NUMBER (\%) } \\
\text { OR MEDIAN } \\
\text { (IQR RANGE) }\end{array}$ \\
\hline \multicolumn{2}{|l|}{ Demographics } \\
\hline Age & 59 (IQR: 51-63) \\
\hline Male & $79(62 \%)$ \\
\hline \multicolumn{2}{|l|}{ Risk factors } \\
\hline Cirrhosis & $6(5 \%)$ \\
\hline COPD & $10(8 \%)$ \\
\hline Corticosteroids before hospitalisation & $11(9 \%)$ \\
\hline Diabetes mellitus & $19(15 \%)$ \\
\hline Haematological malignancy & $19(15 \%)$ \\
\hline Haematological transplant & $5(4 \%)$ \\
\hline Immunosuppression & $32(25 \%)$ \\
\hline Neutropenia & $12(9 \%)$ \\
\hline Previously healthy & $36(28 \%)$ \\
\hline Solid organ malignancy & $11(9 \%)$ \\
\hline Solid organ transplant & $10(8 \%)$ \\
\hline \multicolumn{2}{|l|}{ Aspergillosis classification } \\
\hline Proven & $40(31 \%)$ \\
\hline EORTC Probable & $31(24 \%)$ \\
\hline AsplCU Putative & $31(24 \%)$ \\
\hline Unclassifiable & $47(37 \%)$ \\
\hline \multicolumn{2}{|l|}{ Influenza type } \\
\hline Influenza A & $111(87 \%)$ \\
\hline $\mathrm{A}, \mathrm{H} 1 \mathrm{~N} 1$ & $65(59 \%)^{\mathrm{a}}$ \\
\hline $\mathrm{A}, \mathrm{H} 3$ & $5(4 \%)^{a}$ \\
\hline A, not specified & $41(37 \%)^{\mathrm{a}}$ \\
\hline Influenza B & $12(9 \%)$ \\
\hline Not specified & $5(4 \%)$ \\
\hline \multicolumn{2}{|l|}{ Aspergillosis diagnostics* } \\
\hline BAL culture positive & $46(36 \%)$ \\
\hline 1-3- $\beta$-D-glucan positive & $5(4 \%)$ \\
\hline Computed Tomography & $37(29 \%)$ \\
\hline EORTC defined lesions & $13(35 \%)^{b}$ \\
\hline
\end{tabular}




\begin{tabular}{|l|l|}
\hline Atypical lesions & \multicolumn{1}{|c|}{$24(65 \%)^{\mathrm{b}}$} \\
\hline Galactomannan BAL positive & $39(31 \%)$ \\
\hline Galactomannan serum positive & $60(47 \%)$ \\
\hline Lung biopsy & $20(17 \%)$ \\
\hline Necropsy & $23(18 \%)$ \\
\hline PCR positive & $4(3 \%)$ \\
\hline Sputum culture positive & $43(34 \%)$ \\
\hline Tracheobronchitis & $19(15 \%)$ \\
\hline Time from influenza diagnosis to Aspergillus diagnosis (days) & $5($ IQR: $2-11.5)$ \\
\hline Therapy & \multicolumn{1}{|c|}{$1(1 \%)^{\mathrm{d}}$} \\
\hline Antiviral treatment & $88(69 \%)$ \\
\hline Not specified & $3(93 \%)^{\mathrm{d}}$ \\
\hline Oseltamivir only & \multicolumn{1}{c|}{$2(2 \%)^{\mathrm{d}}$} \\
\hline Oseltamivir and peramivir & $114(89 \%)$ \\
\hline Oseltamivir and zanamivir & $61(48 \%)$ \\
\hline Antifungal treatment & \\
\hline Corticosteroids during hospitalisation & $100(78 \%)$ \\
\hline Outcome & $14(11 \%)$ \\
\hline Mechanical ventilation & $73(57 \%)$ \\
\hline ECMO & $21($ IQR: $13-26.5)$ \\
\hline Mortality &
\end{tabular}


Table 2: Overview of published IAA cases from case reports and case series in the period 1952 - June 2018, listed per country [5-34,35",36-50,51",52",53-54].

\begin{tabular}{|c|c|c|c|c|}
\hline CONTINENT & COUNTRY & $\begin{array}{l}\text { PUBLICATIONS } \\
(n=50)\end{array}$ & YEAR OF PUBLICATION & $\begin{array}{c}\text { IAA } \\
\text { CASES } \\
\text { PER } \\
\text { COUNTRY }\end{array}$ \\
\hline \multicolumn{4}{|l|}{ Africa } & $n=1$ \\
\hline & South-Africa & 1 & 1985 & 1 \\
\hline \multicolumn{4}{|l|}{ Asia } & $n=36$ \\
\hline & Japan & 6 & $1992,1999,2001,2005-2007$ & 7 \\
\hline & Korea & 5 & $2012-2014$ & 5 \\
\hline & Taiwan & 4 & 2013,2017 & 24 \\
\hline \multicolumn{4}{|l|}{ Australia } & $n=1$ \\
\hline & Australia & 1 & 1999 & 1 \\
\hline \multicolumn{4}{|l|}{ Europe } & $n=68$ \\
\hline & Belgium & 2 & 2012,2017 & 10 \\
\hline & France & 3 & $1999,2011,2012$ & 7 \\
\hline & Germany & 2 & 2012,2013 & 6 \\
\hline & Ireland & 1 & 2018 & 2 \\
\hline & Italy & 1 & 2011 & 1 \\
\hline & Poland & 1 & 2011 & 1 \\
\hline & Spain & 3 & 1996, 2011, 2012 & 8 \\
\hline & Switzerland & 2 & 1985,2016 & 2 \\
\hline & The Netherlands & 3 & $2012,2015,2017$ & 26 \\
\hline & United Kingdom & 5 & 1952, 1980, 1982, 1983, 1991 & 5 \\
\hline \multicolumn{4}{|c|}{ North America } & $n=22$ \\
\hline & $\begin{array}{r}\text { United States of } \\
\text { America }\end{array}$ & 10 & $1979,2005,2010-2012,2015-2018$ & 22 \\
\hline
\end{tabular}


1. World Health Organization. WHO guidelines for pharmacological management of pandemic (H1N1) 2009 influenza and other influenza viruses. Geneva, Switzerland, 2010.

2. W.I.V. Influenza [Internet]. Wetenschappelijk Instituut Volksgezondheid; 2017 [Available from: https://www.wiv-isp.be/nl/gezondheidsonderwerpen/influenza-0.

3. McCullers JA. The co-pathogenesis of influenza viruses with bacteria in the lung. Nat Rev Microbiol 2014; 12:252-262

4. Schauwvlieghe AFAD, Rijnders BJ, Philips N, et al. Invasive aspergillosis in patients admitted to the ICU with severe influenza. Article accepted for publication in Lancet Resp Med. 2018; (in press).

- Largest retrospective study showing that influenza is an independent risk factor for invasive aspergillosis.

5. Abbott JD, Fernando HV, Gurling K, Meade BW. Pulmonary aspergillosis following post-influenzal bronchopneumonia treated with antibiotics. Br Med J 1952; 1:523-525.

6. Fischer JJ, Walker DH. Invasive pulmonary aspergillosis associated with influenza. JAMA 1979; 241:1493-1494.

7. Jariwalla AG, Smith AP, Melville-Jones G. Necrotising aspergillosis complicating fulminating viral pneumonia. Thorax 1980; 35:215-216.

8. McLeod DT, Milne $U$, Seaton A. Successful treatment of invasive pulmonary aspergillosis complicating influenza A. Br Med J 1982; 285:1166-1167.

9. Horn CR, Wood NC, Hughes JA. Invasive aspergillosis following post-influenzal pneumonia. Br J Dis Chest 1983; 77:407-410.

10. Urban P, Chevrolet JC, Schifferli J, et al. [Invasive pulmonary aspergillosis associated with an acute influenza virus infection]. Rev Mal Respir 1985; 2:255-257.

11. Lewis $M$, Kallenbach J, Ruff $P$, et al. Invasive pulmonary aspergillosis complicating influenza $A$ pneumonia in a previously healthy patient. Chest 1985; 87:691-693.

12. Hovenden JL, Nicklason F, Barnes RA. Invasive pulmonary aspergillosis in nonimmunocompromised patients. Br Med J 1991; 302:583-584.

13. Kobayashi O, Sekiya M, Saitoh H. [A case of invasive broncho-pulmonary aspergillosis associated with influenza A (H3N2) infection]. Nihon Kyobu Shikkan Gakkai Zasshi 1992; 30:1338-1344.

14. Alba D, Gomez-Cerezo J, Cobo J, et al. [Invasive pulmonary aspergillosis associated with influenza virus]. An Med Interna 1996; 13:34-36.

15. Boots RJ, Paterson DL, Allworth AM, Faoagali JL. Successful treatment of post-influenza pseudomembranous necrotising bronchial aspergillosis with liposomal amphotericin, inhaled amphotericin B, gamma interferon and GM-CSF. Thorax 1999; 54:1047-1049.

16. Funabiki $Y$, Ishii $K$, Kusaka $S$, et al. [Aspergillosis following influenza $A$ infection]. Nihon Ronen Igakkai Zasshi 1999; 36:274-278.

17. Vandenbos F, Mondain-Miton V, Roger PM, et al. [Invasive pulmonary aspergillosis during influenza: a fortuitous association?]. Presse Med 1999; 28:1755.

18. Matsushima $H$, Takayanagi $N$, Ubukata $M$, et al. [Invasive pulmonary aspergillosis following influenza A infection]. Nihon Kokyuki Gakkai Zasshi 2001; 39:672-677.

19. Hasejima N, Yamato K, Takezawa S, et al. Invasive pulmonary aspergillosis associated with influenza B. Respirol 2005; 10:116-119.

20. Lee FE, Daigle CC, Urban MA, et al. Fever and progressive respiratory failure in three elderly family members. Chest 2005; 128:1863-1867.

21. Sugino $\mathrm{K}$, Homma S, Takaya $\mathrm{H}$, et al. [Fatal invasive pulmonary aspergillosis triggered by influenza $B$ virus infection in an individual with idiopathic pulmonary fibrosis]. Nihon Kokyuki Gakkai Zasshi 2006; 44:207-214.

22. Ohnishi $T$, Andou K, Kusumoto $S$, et al. [Two cases of successfully treated invasive pulmonary aspergillosis following influenza virus infection]. Nihon Kokyuki Gakkai Zasshi 2007; 45:349-355.

23. Lat A, Bhadelia N, Miko B, et al. Invasive aspergillosis after pandemic (H1N1) 2009. Emerg Infect Dis 2010; 16:971-973. 
24. Helbig G, Wozniczka K, Wieczorkiewicz A, et al. Irreversible marrow aplasia after single course of 2-chlorodeoxyadenosine for hairy cell leukaemia preceding by A pandemic 2009-H1N1-associated pneumonia. Med Oncol 2011; 28:1601-1603.

25. Adalja AA, Sappington PL, Harris SP, et al. Isolation of Aspergillus in three 2009 H1N1 influenza patients. Influenza Other Respir Viruses 2011; 5:225-229.

26. Carfagna $P$, Brandimarte $F$, Caccese $R$, et al. Occurrence of influenza $A(H 1 N 1) v$ infection and concomitant invasive pulmonary aspergillosis in a patient with chronic obstructive pulmonary disease. Mycoses 2011; 54:549-525.

27. Garcia-Vidal C, Barba $P$, Arnan $M$, et al. Invasive aspergillosis complicating pandemic influenza $A$ (H1N1) infection in severely immunocompromised patients. Clin Infect Dis 2011; 53:e16-19.

28. Passouant $\mathrm{O}$, Mateu $\mathrm{P}$, Commandini $\mathrm{M}$, et al. Pulmonary aspergillosis in non-immunocompromised patient with acute respiratory distress syndrome during A (H1N1) infection. Ann Fr Anesth Réanim 2011; 30:e75-e76.

29. Kim SH, Kim MN, Lee SO, et al. Fatal pandemic influenza A/H1N1 infection complicated by probable invasive pulmonary aspergillosis. Mycoses 2012; 55:189-192.

30. Bagdasarian N, Smith J, Chenoweth C. Invasive pulmonary aspergillosis in patients with 2009 H1N1 influenza infection. Infect Dis Clin Pract 2012; 20:422-424.

31. Guervilly $C$, Roch A, Ranque $S$, et al. A strategy based on galactomannan antigen detection and PCR for invasive pulmonary aspergillosis following influenza A (H1N1) pneumonia. J Infect 2012; 65:470473.

32. Hoyo-Ulloa I, Cobos-Trigueros N, Puig-de la Bellacasa J, Martinez-Martinez JA. Influenza A (H1N1) complicated by invasive aspergillosis in non-severely immunocompromised patients. Enferm Infecc Microbiol Clin 2012; 30:583-584.

33. Vehreschild JJ, Brockelmann PJ, Bangard C, et al. Pandemic 2009 influenza A(H1N1) virus infection coinciding with invasive pulmonary aspergillosis in neutropenic patients. Epidemiol Infect 2012; 140:1848-1852.

34. Otterspoor LC, Smit FH, van Laar TJ, et al. Prolonged use of extracorporeal membrane oxygenation combined with prone positioning in patients with acute respiratory distress syndrome and invasive aspergillosis. Perfusion 2012; 27:335-337.

35. Wauters J, Baar I, Meersseman P, et al. Invasive pulmonary aspergillosis is a frequent complication of critically ill H1N1 patients: a retrospective study. Intensive Care Med 2012; 38:1761-1768.

- First case series indicating that invasive aspergillosis is a frequent complication of severe influenza 36. Kim MJ, Kim MK, Kang CK, et al. A case of acute cerebral aspergillosis complicating influenza A/H1N1pdm 2009. Infect Chemother 2013; 45:225-229.

37. Kwon OK, Lee MG, Kim HS, et al. Invasive pulmonary aspergillosis after influenza a infection in an immunocompetent patient. Tuberc Respir Dis (Seoul) 2013; 75:260-263.

38. Toh H-S, Jiang M-Y, Tay H-T. Invasive pulmonary aspergillosis in severe complicated influenza A. J Formos Med Assoc 2013; 112:810-811.

39. Yildirim $Y$, Pecha S, Sill B, et al. Severe bacterial superinfection based on influenza A (H1N1) pneumonia in a heart-lung transplant recipient. Thorac Cardiovasc Surg 2013; 61:255-257.

40. Lee JY, Joo EJ, Yeom JS, et al. Aspergillus tracheobronchitis and influenza A co-infection in a patient with AIDS and neutropenia. Infect Chemother 2014; 46:209-215.

41. Park DW, Yhi JY, Koo G, et al. Fatal clinical course of probable invasive pulmonary aspergillosis with influenza B infection in an immunocompetent patient. Tuberc Respir Dis (Seoul) 2014; 77:141-144.

42. Alshabani K, Haq A, Miyakawa R, et al. Invasive pulmonary aspergillosis in patients with influenza infection: report of two cases and systematic review of the literature. Expert Rev Respir Med 2015; 9:89-96.

43. Kolwijck E, Scheper $\mathrm{H}$, Beuving J, et al. [Invasive pulmonary aspergillosis in influenza]. Ned Tijdschr Geneeskd 2015; 159:A7431.

44. Pietsch U, Muller-Hocker C, Enzler-Tschudy A, Filipovic M. Severe ARDS in a critically ill influenza patient with invasive pulmonary aspergillosis. Intens Care Med 2016; 42:1632-1633. 
45. Crum-Cianflone NF. Invasive aspergillosis associated with severe influenza infections. Open Forum Infect Dis 2016; 3:ofw171.

46. Nulens EF, Bourgeois MJ, Reynders MB. Post-influenza aspergillosis, do not underestimate influenza B. Infect Drug Resist 2017; 10:61-67.

47. Su P-A, Yu W-L. Failure of extracorporeal membrane oxygenation to rescue acute respiratory distress syndrome caused by dual infection of influenza $A(H 1 N 1)$ and invasive pulmonary aspergillosis. J Formos Med Assoc 2017; 116:563-564.

48. Hou K, Sutherland A. Bacterial pneumonia with influenza coinfection complicated by aspergillosis, ards, and septic shock in a cirrhotic. Am J Respir Crit Care Med 2017;195.

49. Ku YH, Chuang YC, Yu WL. Postinfluenza A(H3N2) refractory invasive pulmonary aspergillosis. J Formos Med Assoc 2017; 116:404-405.

50. Ku YH, Chan KS, Yang CC, et al. Higher mortality of severe influenza patients with probable aspergillosis than those with and without other coinfections. J Formos Med Assoc 2017; 116:660-670. 51. van de Veerdonk FL, Kolwijck E, Lestrade PP, et al. Influenza-associated aspergillosis in critically ill patients. Am J Respir Crit Care Med 2017.

- Large case series showing high incidence and mortality of critically ill influenza patients with secondary aspergillosis.

52. Shah MM, Hsiao El, Kirsch CM, et al. Invasive pulmonary aspergillosis and influenza co-infection in immunocompetent hosts: case reports and review of the literature. Diagn Microbiol Infect Dis 2018; 91:147-152.

- Review of epidemiology and risk factors for influenza-associated aspergillosis.

53. Talento AF, Dunne K, Murphy N, et al. Post-influenzal triazole-resistant aspergillosis following allogeneic stem cell transplantation. Mycoses 2018; Mar 23. doi: 10.1111/myc.12770. [Epub ahead of print].

54. Ajmal S, Mahmood M, Abu Saleh O, et al. Invasive fungal infections associated with prior respiratory viral infections in immunocompromised hosts. Infection 2018; Apr 7. doi: 10.1007/s15010-018-11380 . [Epub ahead of print].

55. Martin-Loeches I, J Schultz M, Vincent JL, et al. Increased incidence of co-infection in critically ill patients with influenza. Intensive Care Med 2017; 43:48-58.

56. Rodriguez-Goncer I, Thomas S, Foden P, et al. Invasive pulmonary aspergillosis is associated with adverse clinical outcomes in critically ill patients receiving veno-venous extracorporeal membrane oxygenation. Eur J Clin Microbiol Infect Dis 2018; 37:1251-1257.

57. Cavayas YA, Yusuff H, Porter R. Fungal infections in adult patients on extracorporeal life support. Crit Care 2018; 22:98.

- Large retrospective analysis of an international ECMO database identifying influenza as a risk factor for positive Aspergillus cultures in ECMO patients.

58. Paules C, Subbarao K. Influenza. Lancet 2017; 390:697-708.

59. Reddy KP, Bajwa EK, Parker RA, et al. Relationship between upper respiratory tract influenza test result and clinical outcomes among critically ill influenza patients. Open Forum Infect Dis 2016; 3:ofw023.

60. Patterson TF, Thompson GR, 3rd, Denning DW, et al. Practice guidelines for the diagnosis and management of aspergillosis: 2016 Update by the Infectious Diseases Society of America. Clin Infect Dis 2016; 63:e1-e60.

61. Krenke R, Grabczak EM. Tracheobronchial manifestations of Aspergillus infections. ScientificWorldJournal 2011; 11:2310-2329.

62. Eigl S, Prattes J, Lackner M, et al. Multicenter evaluation of a lateral-flow device test for diagnosing invasive pulmonary aspergillosis in ICU patients. Crit Care 2015; 19:178.

63. Ullmann AJ, Aguado JM, Arikan-Akdagli S, et al. Diagnosis and management of Aspergillus diseases: executive summary of the 2017 ESCMID-ECMM-ERS guideline. Eur J Clin Microbiol Infect Dis 2018; 24 Suppl 1:e1-e38. 
64. Buil JB, van der Lee HAL, Rijs A, et al. Single-center evaluation of an agar-based screening for azole resistance in Aspergillus fumigatus by using VIPcheck. Antimicrob Agents Chemother 2017; 61. pii:e01250-17.

65. Lestrade P, Bentvelsen RG, Schauwvlieghe AFAD, et al. Voriconazole resistance and mortality in invasive aspergillosis: a multicenter retrospective cohort study. Clin Infect Dis 2018; (in press).

66. Buil JB, Zoll J, Verweij PE, et al. Molecular detection of azole-resistant Aspergillus fumigatus in clinical samples. Front Microbiol 2018; 9:515.

67. Chong GM, van der Beek MT, von dem Borne PA, et al. PCR-based detection of Aspergillus fumigatus Cyp51A mutations on bronchoalveolar lavage: a multicentre validation of the AsperGenius assay ${ }^{\circledR}$ in 201 patients with haematological disease suspected for invasive aspergillosis. J Antimicrob Chemother 2016; 71:3528-3535.

68. Buchner J, Mazzeffi M, Kon Z, et al. Single-center experience with venovenous ECMO for influenzarelated ARDS. J Cardiothorac Vasc Anesth 2018; 32:1154-1159.

69. Pasi $P$, Mike C. ECDC expert opinion on efficacy and effectiveness of neuraminidase inhibitors published for public consultation. Influenza Other Respir Viruses 2016; 10:152-153.

70. European Medical Agency. Alpivab : EPAR - Public assessment report. London: European Medicines Agency; 2018. p. 108.

71. Rodriguez A, Diaz E, Martin-Loeches I, et al. Impact of early oseltamivir treatment on outcome in critically ill patients with 2009 pandemic influenza A. J Antimicrob Chemother 2011; 66:1140-1149.

72. Richard F, Mahieu R, Gullou-Guillemette HL, et al. Prognosis factors of severe influenza in ICU and introduction delay of oseltamivir. Annals of Intensive Care 2017; 7:21-22.

73. Hernu R, Chroboczek T, Madelaine T, et al. Early oseltamivir therapy improves the outcome in critically ill patients with influenza: a propensity analysis. Intensive Care Med 2018; 44:257-260.

74. Flannery AH, Thompson Bastin ML. Oseltamivir dosing in critically ill patients with severe influenza. Ann Pharmacother 2014; 48:1011-1018.

75. Kiser T, Burnham E, Ho M, et al. 660: Extended-duration versus standard-duration oseltamivir in critically ill patients with influenza. Crit Care Med 2018; 46:316.

76. Network SEAIDCR. Effect of double dose oseltamivir on clinical and virological outcomes in children and adults admitted to hospital with severe influenza: double blind randomised controlled trial. $\mathrm{Br}$ Med J 2013; 346:f3039.

77. Lee N, Hui DS, Zuo Z, et al. A prospective intervention study on higher-dose oseltamivir treatment in adults hospitalized with influenza a and B infections. Clin Infect Dis 2013; 57:1511-1519.

78. Kumar A. Viral clearance with standard or triple dose oseltamivir therapy in critically ill patients with pandemic (H1N1) 2009 influenza. 53rd Interscience Conference on Antimicrobial Agents and Chemotherapy; September 10-13; Denver, CO 2013. p. B-1470.

79. Kiser T, Burnham E, Ho M, et al. 656: Evaluation of high-dose versus standard-dose oseltamivir in critically ill patients with influenza. Crit Care Med 2018; 46:314.

- Retrospective analysis of 19,892 critically ill influenza patients receiving either high-dose or standard-dose oseltamivir, showing no clinical benefit of high-dose therapy.

80. Welch SC, Lam SW, Neuner EA, et al. High-dose versus standard dose oseltamivir for treatment of severe influenza in adult intensive care unit patients. Intensive Care Med 2015; 41:1365-1366.

81. Kmietowicz Z. WHO downgrades oseltamivir on drugs list after reviewing evidence. BMJ 2017; 357:j2841.

82. Ariano RE, Sitar DS, Zelenitsky SA, et al. Enteric absorption and pharmacokinetics of oseltamivir in critically ill patients with pandemic (H1N1) influenza. CMAJ 2010; 182:357-363.

83. Eyler RF, Heung M, Pleva M, et al. Pharmacokinetics of oseltamivir and oseltamivir carboxylate in critically ill patients receiving continuous venovenous hemodialysis and/or extracorporeal membrane oxygenation. Pharmacotherapy 2012; 32:1061-1069.

84. Mulla H, Peek GJ, Harvey C, et al. Oseltamivir pharmacokinetics in critically ill adults receiving extracorporeal membrane oxygenation support. Anaesth Intensive Care 2013; 41:66-73. 
85. Lemaitre F, Luyt CE, Roullet-Renoleau F, et al. Impact of extracorporeal membrane oxygenation and continuous venovenous hemodiafiltration on the pharmacokinetics of oseltamivir carboxylate in critically ill patients with pandemic (H1N1) influenza. Ther Drug Monit 2012; 34:171-175.

86. 39th ESCP European symposium on clinical pharmacy \& 13th SFPC congress: clinical pharmacy at the front line of innovations. 21-23 October 2010, Lyon, France. Int J Clin Pharm 2011; 33:285-467.

87. Kromdijk W, Sikma MA, van den Broek MPH, et al. Pharmacokinetics of oseltamivir carboxylate in critically ill patients: each patient is unique. Intensive Care Med 2013; 39:977-978.

88. Eschenauer GA, Lam SW. Supratherapeutic oseltamivir levels during continuous dialysis: an expected risk. Intensive Care Med 2011; 37:371.

89. Ison MG. Antiviral Treatments. Clin Chest Med 2017; 38:139-153.

90. Naesens L, Stevaert A, Vanderlinden E. Antiviral therapies on the horizon for influenza. Curr Opin Pharmacol 2016; 30:106-115.

91. Herbrecht R, Denning DW, Patterson TF, et al. Voriconazole versus amphotericin B for primary therapy of invasive aspergillosis. N Engl J Med 2002; 347:408-415.

92. Spriet I, Annaert P, Meersseman P, et al. Pharmacokinetics of caspofungin and voriconazole in critically ill patients during extracorporeal membrane oxygenation. J Antimicrob Chemother 2009; 63:767-770.

93. Ruiz S, Papy E, Da Silva D, et al. Potential voriconazole and caspofungin sequestration during extracorporeal membrane oxygenation. Intensive Care Med 2009; 35:183-184.

94. Li TY, Liu W, Chen K,et al. The influence of combination use of CYP450 inducers on the pharmacokinetics of voriconazole: a systematic review. J Clin Pharm Ther 2017; 42:135-146.

95. Andes D, Azie N, Yang H, et al. Drug-drug interaction associated with mold-active triazoles among hospitalized patients. Antimicrob Agents Chemother 2016; 60:3398-3406.

96. Muilwijk EW, Dekkers BGJ, Henriet SSV, et al. Flucloxacillin results in suboptimal plasma voriconazole concentrations. Antimicrob Agents Chemother 2017; 61.pii: e00915-17

- Small case series highlighting the need for therapeutic drug monitoring when coadministering flucloxacillin and voriconazole.

97. Maertens JA, Raad II, Marr KA, et al. Isavuconazole versus voriconazole for primary treatment of invasive mould disease caused by Aspergillus and other filamentous fungi (SECURE): a phase 3, randomised-controlled, non-inferiority trial. Lancet 2016; 387:760-769.

98. Ledoux MP, Toussaint E, Denis J, Herbrecht R. New pharmacological opportunities for the treatment of invasive mould diseases. J Antimicrob Chemother 2017; 72(suppl_1):i48-i58.

99. Marty FM, Ostrosky-Zeichner L, Cornely OA, et al. Isavuconazole treatment for mucormycosis: a single-arm open-label trial and case-control analysis. Lancet Infect Dis 2016; 16:828-837.

100. Stott KE, Hope WW. Therapeutic drug monitoring for invasive mould infections and disease: pharmacokinetic and pharmacodynamic considerations. J Antimicrob Chemother 2017; 72(suppl_1):i12-i8.

101. Bassetti M, Carnelutti A, Righi E. Issues in the management of invasive pulmonary aspergillosis in non-neutropenic patients in the intensive care unit: A role for isavuconazole. IDCases 2018; 12:7-9.

102. Resendiz Sharpe A, Lagrou K, Meis JF, Chowdhary A, Lockhart SR, Verweij PE. Triazole resistance surveillance in Aspergillus fumigatus. Med Mycol 2018; 56(suppl_1):83-92.

- Review of current knowledge on triazole resistance, including worldwide epidemiology and expert opinion.

103. van Paassen J, Russcher A, In 't Veld-van Wingerden AW, et al. Emerging aspergillosis by azoleresistant Aspergillus fumigatus at an intensive care unit in the Netherlands, 2010 to 2013. Euro Surveill 2016; 21.

104. Verweij PE, Ananda-Rajah M, Andes D, et al. International expert opinion on the management of infection caused by azole-resistant Aspergillus fumigatus. Drug Resist Updat 2015; 21-22:30-40.

105. Bassetti M, Garnacho-Montero J, Calandra T, et al. Intensive care medicine research agenda on invasive fungal infection in critically ill patients. Intens Care Med 2017; 43:1225-1238.

106. Nivoix $Y$, Velten $M$, Letscher-Bru $V$, et al. Factors associated with overall and attributable mortality in invasive aspergillosis. Clin Infect Dis 2008; 47:1176-1184. 
107. Thompson BT, Chambers RC, Liu KD. Acute Respiratory Distress Syndrome. N Engl J Med 2017; 377:1904-1905.

108. Cooper MS, Stewart PM. Corticosteroid insufficiency in acutely ill patients. N Engl J Med 2003; 348:727-734.

109. Venkatesh B, Finfer S, Avni T, et al. Adjunctive glucocorticoid therapy in patients with septic shock. N Engl J Med 2018; 378:797-808.

110. Stern A, Skalsky K, Avni T, et al. Corticosteroids for pneumonia. Cochrane database Syst Rev 2017; 12:Cd007720.

- Systematic review on the use of systemic corticosteroids in pneumonia-patients, showing improved morbidity and mortality with hyperglycemia as major side-effect.

111. Prina E, Ceccato A, Torres A. New aspects in the management of pneumonia. Crit Care 2016; 20:267.

112. Giannella M, Alonso M, Garcia de Viedma D, et al. Prolonged viral shedding in pandemic influenza A(H1N1): clinical significance and viral load analysis in hospitalized patients. Eur J Clin Microbiol Infect Dis 2011; 17:1160-1165.

113. Nedel WL, Nora DG, Salluh JIF, et al. Corticosteroids for severe influenza pneumonia: A critical appraisal. World J Crit Care Med 2016; 5:89-95.

114. Delaney JW, Pinto R, Long J, et al. The influence of corticosteroid treatment on the outcome of influenza A(H1N1pdm09)-related critical illness. Crit Care 2016; 20:75.

115. Meersseman W, Lagrou K, Maertens J, Van Wijngaerden E. Invasive aspergillosis in the intensive care unit. Clin Infect Dis 2007; 45:205-216.

116. Lionakis MS, Kontoyiannis DP. Glucocorticoids and invasive fungal infections. Lancet 2003; 362:1828-1838.

117. Bassetti M, Bouza E. Invasive mould infections in the ICU setting: complexities and solutions. J Antimicrob Chemother 2017; 72(suppl_1):i39-i47.

- Review addressing the problems of diagnosing and treating IPA in the intensive care unit.

118. Bermejo-Martin JF, Martin-Loeches I, Rello J, et al. Host adaptive immunity deficiency in severe pandemic influenza. Crit Care 2010; 14:R167.

119. Walther $E, X u Z$, Richter $M$, et al. Dual acting neuraminidase inhibitors open new opportunities to disrupt the lethal synergism between Streptococcus pneumoniae and influenza virus. Front Microbiol 2016; 7:357.

120. Dewi IMW, Cunha C, Vanderbeke L, et al. Oseltamivir affects host defense against invasive pulmonary aspergillosis. Abstract presented at ECCMID 2018; 2018 April 2018; Madrid.

- Conference abstract showing effect of oseltamivir on host susceptibility to Aspergillus in vivo and in vitro. 